\section{(6) OPEN ACCESS}

\title{
Complications to invasive epilepsy surgery workup with subdural and depth electrodes: a prospective population-based observational study
}

\author{
Emelie Hedegärd, ${ }^{1}$ Johan Bjellvi, ${ }_{1}^{1}$ Anna Edelvik, ${ }^{1}$ Bertil Rydenhag, ${ }^{1}$ Roland Flink, ${ }^{2}$ \\ Kristina Malmgren ${ }^{1}$
}

1 Epilepsy Research Group, Institute of Neuroscience and Physiology, Sahlgrenska Academy at Gothenburg University, Göteborg, Sweden ${ }^{2}$ Department of Clinical Neurophysiology, Uppsala University Hospital, Uppsala, Sweden

\section{Correspondence to} Professor Kristina Malmgren, Epilepsy Research Group, Institute of Neuroscience and Physiology, Section of Clinical neuroscience and

Rehabilitation Per Dubbsgatan $141 \mathrm{tr}$, Sahlgrenska Academy at Gothenburg University, 41345 Göteborg Sweden; Kristina.malmgren@neuro.gu. se

Received 2 August 2013 Revised 11 November 2013 Accepted 13 November 2013 Published Online First 29 November 2013
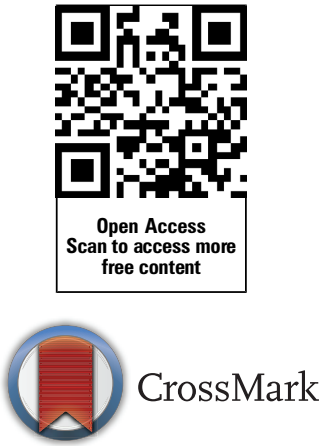

To cite: Hedegärd $\mathrm{E}$, Bjellvi J, Edelvik $A$, et al. $J$ Neurol Neurosurg Psychiatry 2014;85: 716-720.

\begin{abstract}
Objective In some patients who undergo presurgical workup for drug-resistant epilepsy invasive seizure monitoring is needed to define the seizure onset zone and delineate eloquent cortex. Such procedures carry risks for complications causing permanent morbidity and even mortality. In this study, prospective data on complications in a national population-based sample were analysed.

Design Complication data from the prospective Swedish National Epilepsy Surgery Register were analysed for 271 patients in whom therapeutic surgery was preceded by invasive monitoring 1996-2010.
\end{abstract} Results Complications occurred in 13/271 patients (4.8\%). Subdural grids carried the highest risk of complications (7.4\%). There was no surgical mortality or permanent morbidity. Subdural haematomas were most common $(n=7)$ followed by epidural haematomas $(n=3)$. Valproate treatment and having a haematoma was associated with an OR of 1.53 ( $\mathrm{Cl} 0.38$ to 6.12 ) compared to having a haematoma without valproate treatment. Having a complication during invasive monitoring was associated with a significant OR of 6.27 (CI 1.32 to 29.9) of also having a complication at therapeutic surgery compared to the risk of having a complication only at surgery.

Conclusions In this prospective population-based epilepsy surgery series, the most common complications were haematomas, and subdural grids carried the highest risk. Close supervision and rapid interventions led to avoidance of permanent morbidity. The clinical implications of the slightly increased risk of haematomas with valproate treatment needs further investigation as does the finding of an increased risk for complications at epilepsy surgery for patients who had a complication during invasive monitoring.

\section{INTRODUCTION}

Epilepsy surgery is an established treatment in selected patients with drug-resistant epilepsy resulting in seizure freedom or a significant reduction of seizure frequency in the majority of operated patients. ${ }^{1-3}$

A detailed clinical assessment is critical before a decision about surgery can be made in order to localise the seizure onset zone. For many patients, a non-invasive presurgical workup is satisfactory. However, especially in MRI-negative patients, invasive seizure monitoring is required for localising the seizure onset zone and also for delineating the area of planned resection against eloquent cortex. ${ }^{4}$

Despite the potential benefits of the invasive workup, they have to outweigh the risks related to the neurosurgical procedure. The rates of reported complications related to invasive monitoring differ widely between studies, from no complications at all ${ }^{5}$ up to $26.3 \% .^{6}$ The reasons for these varying proportions may be related to the definitions of complications and to study methodology (eg, if data are obtained retrospectively or prospectively).

Different classifications of complications have been suggested. Many reports use the terms transient and permanent, surgical and neurological complications. ${ }^{78}$ Additionally, severity scales from 1 to $4,{ }^{9}$ or from 1 to 5 have been used. ${ }^{4}$ In the Swedish National Epilepsy Surgery Register all complications related to diagnostic or therapeutic epilepsy surgery procedures are reported and are graded as major or minor. ${ }^{10}$

A few studies have evaluated the usefulness and safety versus the risks associated with implantation of intracranial electrodes, and the benefits of invasive workup for epilepsy surgery in general seem to outweigh the risks. ${ }^{11} 12$

Increased number of electrodes and longer duration of monitoring has been shown to be associated with increased frequency of adverse events. ${ }^{13}$ Other aspects not explored include age and medication. Higher age was shown to be associated with an increased risk for complications at epilepsy surgery in an earlier Swedish national study ${ }^{10}$ and, therefore, it would be of interest to investigate whether the risk of complications related to invasive monitoring is also age related. Valproate treatment may induce thrombocytopenia which seems to be dose dependent with a negative correlation between valproate trough levels and platelet counts. ${ }^{14}$ Although several studies have found no indication that patients on valproate treatment are at an increased risk of surgical bleeding complications, ${ }^{15} 16$ there is a worry about the possible risk, and some epilepsy surgeons do not operate patients who are on valproate treatment.

The aim of the current study was to analyse data from the prospective population-based Swedish National Epilepsy Surgery Register 1996-2010, in order to describe the complications related to preoperative invasive investigations with intracranial electrodes. Secondary aims were to explore if the risk of complications is age related, if there was a 
higher risk of haemorrhagic complications in patients who were treated with valproate, and whether complications occurring during invasive monitoring is a risk factor for complications at therapeutic surgery.

\section{PATIENTS AND METHODS}

Every epilepsy surgery procedure in Sweden is reported to the Swedish National Epilepsy Surgery Register. The register includes data on all patients who have been operated in Sweden since 1990. It was initiated by the Swedish Board of Health and Welfare as a quality control register, and is controlled by the Swedish Data Inspection Board. All patients whose data are included in the register have given their informed consent. The validity of the data collected from the centres is regularly checked by intrinsic checkpoints within the database and by external revision. Two epilepsy nurses visit the six centres regularly and compare the data entered into the central database with the original data from the patient files for a random yearly sample of around $25 \%$ of the patients operated at each centre. The items controlled in this external revision include side and site of operation, complications, main histopathological findings and seizure outcome at the two-year follow-up. So far, there have been no mismatches in the reporting on any of these central items. The Regional Board of Medical Ethics at the University of Gothenburg considered this study a quality control study not necessitating informed consent for research.

In this study, we have analysed complications after invasive EEG monitoring in all patients who, prior to surgery, underwent investigations with subdural strips or grids, intracerebral depth electrodes, foramen ovale electrodes or epidural electrodes from January 1996 to December 2010. (During this time period, Stereo-EEG (SEEG) was not performed in Sweden, the depth electrodes used were freely placed most often in conjunction with grid electrodes). Two or more electrode types were often combined, and when reporting complications in relation to electrode type the most complication-prone electrode type has been accounted for. The register does, however, not provide information on the number of electrodes and the duration of monitoring.

Complications are reported as major or minor to the register, and the type of complication is commented upon. Minor complications are defined as those that resolve within 3 months, while major complications affect activities of daily living and last longer than 3 months. Major complications also include any significant neurological deficits, even if they do not affect activities of daily living. ${ }^{10}$

The antiepileptic drug treatment at the presurgical investigation is reported, but not the dosage. The Swedish National Epilepsy Surgery register does not contain information on laboratory variables, such as trough levels of antiepileptic drugs or platelet counts.

Descriptive statistics were used. For comparison between two groups, Fisher's Exact test was used for dichotomous variables. For descriptive purposes ORs with corresponding CIs were calculated. All tests were two-tailed and conducted at 0.05 significance level.

\section{RESULTS}

Between 1 January 1996 and 31 December 2010, 865 patients underwent therapeutic surgical procedures. Seven hundred and ninety-eight of the operations were resections: 523 were temporal lobe resections (TLR) and 142 were frontal lobe resections (FLR). There were 44 parietal lobe resections (PLR), 27 occipital lobe resections (OLR), 19 multilobar resections (MLR) and 43 hemispherectomies. Two hundred and seventy-six of the 865 patients (32\%) underwent diagnostic intracranial monitoring with invasive electrodes prior to the therapeutic surgical procedure. Two hundred and sixty-four of them had a resective procedure later, and there were eight multiple subpial trans-sections, three callosotomies and one exploration. Forty three per cent $(n=61)$ of the patients who eventually had an FLR were invasively monitored, versus $30 \%(n=156)$ of the patients who had a TLR, $57 \%$ of the patients who had a PLR, $30 \%$ of the patients who had an OLR, 63\% of the patients who had an MLR and 5\% of the patients who had a hemispherectomy.

Patient age at surgery ranged from two years to 58 years (median 26 years), 134 (48.6\%) were female and 142 (51.4\%) were male. For five patients, data on complications to the invasive investigation were missing, which is why complication data are reported for 271 patients. For another five patients, data on electrode type was missing, hence, the analysis of electrode type related to surgical complications is limited to 271 patients.

There was no surgical mortality in this series. Also, there were no major complications. Minor complications occurred in 13 of 271 cases $(4.8 \%)$ (table 1$)$.

Haematomas were most common, associated with a $3.7 \%$ risk $(n=10)$. Of these, seven $(2.6 \%)$ were subdural haematomas and three were epidural haematomas (1.1\%). Two patients suffered from wound infections and one from electrode dislocation, representing a complication rate of 0.7 and $0.4 \%$. Individual patient data for all complications occurring during intracranial monitoring are given in table 2 .

Ten per cent $(n=6)$ of the 61 patients who were investigated for an FLR and 4\% $(n=6)$ of the 156 patients investigated for a TLR suffered from complications from the invasive electrode monitoring. In the group of patients who had complications, five out of the six who had frontally located electrodes had a subdural haematoma. Among the patients with complications from temporally located electrodes, the complication types were more varied. There was a slight, non-significant difference in complication rate between female $(3.9 \%, \mathrm{n}=5)$ and male patients $(5.7 \%, \mathrm{n}=8)$.

Among the 108 patients who were monitored with subdural grids, eight (7.4\%) suffered from complications, mostly haematomas. As shown in table 3, grids had the highest complication rate, followed by intracerebral depth electrodes. Two patients in the study had epidural electrodes, and one of them got a subdural haematoma.

When patients were divided into age categories (table 4) there was a difference between the age groups. Children (age 5-18 years) and young adults (age 18-35 years) had complications in 3.2 and $3.6 \%$, respectively, while $8.3 \%$ of adults $\geq 35$ years old had complications. This difference did not reach statistical significance $(\mathrm{p}=0.12)$.

Sixty of the 271 patients $(22 \%)$ in who reports on complications or not had been registered were treated with valproate at

Table 1 Complications related to intracranial monitoring

\begin{tabular}{lr}
\hline Type of complication & $\mathbf{n}(\%)$ \\
\hline Subdural haematoma & $7(2.6)$ \\
Epidural haematoma & $3(1.1)$ \\
Wound infection & $2(0.7)$ \\
Electrode dislocation & $1(0.4)$ \\
Total & $13(4.8)$ \\
\hline
\end{tabular}


Table 2 Individual complications occurring during intracranial monitoring

\begin{tabular}{|c|c|c|c|c|}
\hline $\begin{array}{l}\text { Patient; gender } \\
\text { (age-years) }\end{array}$ & Resection type & Electrode type & Complication type & Comment in the register \\
\hline Female (13) & RFLR & Strips+grids & Subdural haematoma & Haematoma under the grid (evacuated) \\
\hline Male (14) & RFLR & Strips+grids & Subdural haematoma & Haematoma under the grid (evacuated) \\
\hline Male (16) & LTLR & Epidural & Subdural haematoma & No comment \\
\hline Male (18) & LFLR & Grids & Subdural haematoma & Haematoma under the grid (evacuated) \\
\hline Male (20) & LFLR & Strips+grids & Subdural haematoma & Haematoma under the grid \\
\hline Female (34) & RTLR & Strips+intracerebral & Electrode dislocation & A subdural strip electrode was dislocated intraparenchymally \\
\hline Male (36) & LTLR & Strips & Infection & Wound infection \\
\hline Female (36) & RFLR & Grids & Subdural haematoma & Haematoma under the grid \\
\hline Male (39) & LTLR & Strips+grids+intracerebral & Epidural haematoma & Epidural haematoma after implantation of grids (evacuated) \\
\hline Male (40) & MST & Strips & Epidural haematoma & Epidural hematoma in connexion with electrode implantation (evacuated) \\
\hline Female (42) & LTLR & Strips+grids & Subdural haematoma & Haematoma under the grid \\
\hline Female (44) & RTLR & Strips & Infection & Wound infection \\
\hline Male (56) & LFLR & Grids & Epidural haematoma & Epidural haematoma (evacuated) \\
\hline
\end{tabular}

the time of presurgical investigations, alone or in combination with other antiepileptic drugs. Three of the 60 patients on valproate had a bleeding during invasive monitoring (5\%) compared with seven of the 211 patients who were not treated with valproate $(3.3 \%)$. Treatment with valproate and having a bleeding was associated with an OR of 1.53 (95\% CI 0.38 to 6.12) compared to having a bleeding and not being treated with valproate. This association was, however, not significant as shown by the wide CI including 1 .

In the whole Swedish series of 865 therapeutic surgeries 1996-2010, there were 26 major complications (3\%). ${ }^{17}$ A post hoc analysis of a possible relationship between having a complication during invasive monitoring and at surgery was performed. Two of 13 patients (15\%) who had a complication at the invasive monitoring in addition also suffered a major complication related to the therapeutic procedure, compared to 24/ 852 (3\%) patients who had a complication only at therapeutic surgery. Having a complication during invasive monitoring was associated with a significant OR of 6.27 (CI 1.32 to 29.9) of also having a complication at therapeutic surgery compared to the risk of having a complication only at surgery. There was no relationship between the kind of complication (bleeding or other) at the one or the other procedure.

\section{DISCUSSION}

In this series, the rate of haemorrhagic complications $3.6 \%$ of the patients) was comparable with that reported in a recent systematic review concerning adverse events related to invasive

Table 3 Electrode types and complications

\begin{tabular}{|c|c|c|c|}
\hline $\begin{array}{l}\text { Type of invasive } \\
\text { electrodes* }\end{array}$ & n (\%) & $\begin{array}{l}\text { Complications per } \\
\text { electrode type } n(\%)\end{array}$ & Type \\
\hline Grids & $108(40.5)$ & $8(7.4)$ & $6 \mathrm{SH} / 2 \mathrm{EH}$ \\
\hline Intracerebral depth & $14(5.2)$ & $1(7.1)$ & $1 \mathrm{D}$ \\
\hline Foramen ovale & $9(3.4)$ & 0 & - \\
\hline Epidural & $2(0.7)$ & $1(50)$ & $1 \mathrm{SH}$ \\
\hline Strips only & $134(50.2)$ & $3(2.2)$ & $1 \mathrm{EH} / 2 \mathrm{I}$ \\
\hline
\end{tabular}

EEG monitoring with subdural grid electrodes which identified 21 studies including 2542 patients. ${ }^{13}$

In this review, the mean pooled prevalence of haematomas was $4 \%$. The development of subdural haematoma was the most common type of complication $(2.6 \%)$ in our series, more than half the complications were in this category $(n=7)$. The prevalence of subdural haematomas varies widely in the literature and is reported to be common in some studies, whereas, other groups report no subdural haematomas at all in their series. In the abovementioned review, subdural haematomas were documented in 41 of 2356 patients (pooled prevalence 1.7\%). ${ }^{13}$ Three $(1.1 \%)$ of our patients developed epidural haematomas during the invasive EEG monitoring. This is slightly higher than the $0.6 \%$ reported by Arya et al. ${ }^{13}$ Even higher rates have been reported by Hamer et al $(2.5 \%),{ }^{6}$ while several studies report no epidural haematomas at all. ${ }^{18-20}$

Two $(0.7 \%)$ of the patients in our study had wound infections, whereas, there were no intracranial infections. This is a low number compared to other studies. In the review by Arya et al, infections emerged as the most common type of complication with a pooled prevalence of 5.3\% (2.3\% neurologic infections and $3.0 \%$ superficial infections). ${ }^{13}$ Some series also have significantly higher infection rates. ${ }^{9} 21$ In one prospective study, the infection rate was shown to increase if more than 100 electrode contacts were used, if more than ten electrode cables were present, or if electrodes remained implanted for more than 14 days. $^{22}$ Since the Swedish National Epilepsy Surgery Register does not contain data on number of electrode contacts or days, we were not able to analyse the impact of these factors in our series. We hypothesise that one reason we do not have any bone flap or deep infections may be attributed to short surgical

Table 4 Age and complications

\begin{tabular}{lcc}
\hline Age (years) & Patients $(\mathbf{n})$ & Complications $\mathbf{n}(\%)$ \\
\hline$\geq 2-<5$ & 9 & 0 \\
$\geq 5-<18$ & 84 & $3(3.6)$ \\
$\geq 18-<35$ & 94 & $3(3.2)$ \\
$\geq 35$ & 84 & $7(8.3)$ \\
Total & 271 & 13 \\
\hline
\end{tabular}


time $^{23}$ since we, in general, use fewer grids than reported from many centres in complication series.

In our series, grid electrodes caused more complications than other types of electrodes, as eight of 13 affected patients had grid electrodes alone or in combination with other electrode types. This correlation between subdural grids and complications has also been shown by other groups. ${ }^{4} 24$ The reasons for this increased risk are unclear but could be mechanical or haemodynamic (the grid disturbing the CSF circulation). Whether the addition of strips to a subdural grid recording increases the risk of complications cannot be excluded, although strips per se have a low complication rate. Patients who are investigated for frontal lobe seizure onset tend to have more grid electrodes than other patients. This group of patients also suffered from complications more often than other groups in our series, nearly $10 \%$ (6 out of 61 ), and all were haematomas. Next in line were the patients investigated for temporal lobe epilepsy, almost 4\% (6 out of 156) had complications. We assume that the high rate of haemorrhagic complication in patients with frontal lobe seizures was related to the more common use of grids.

There was a (non-significant) trend towards higher risk for complications in patients who were 35 years or more in our series. The association between complications and age has previously been documented in relation to invasive monitoring, resective epilepsy surgery procedures and other neurosurgical procedures, and has been discussed in terms of increasing tissue fragility and affection of the cerebrovascular system with increasing age. ${ }^{6} 1025$ On the other hand, Blauwblomme et al studied adverse events related to invasive EEG monitoring in children and found a very high complication rate, almost half the patients suffered from some kind of complication. This can partly be explained by the fact that in this series a wide range of adverse events was considered as complications, for example, cerebrospinal leakage. ${ }^{9}$ Minor adverse events such as headache, nausea, light fever and tiredness during the first postoperative days can be associated with the implantation of subdural electrodes, but most surgeons consider them as expected effects of the implantation and they are rarely documented.

In the post hoc exploration of a possible relationship between complications during invasive monitoring and at surgery it was notable that two of the 13 patients with complications in our study also suffered from major complications after the therapeutic resection. ${ }^{17}$ Since this association was not explained by bleedings, some other vulnerability may be at play, for which we presently have no explanation. In order to verify and elucidate this finding, prospective multicentre studies of complications during invasive monitoring and in relation to resective surgery, collecting more detailed patient data is needed.

There was no significant association between valproate treatment and bleeding complications during invasive monitoring and, therefore, there is no support for the notion that valproate treatment is an important risk factor for bleedings. However, the OR of 1.53 implies that there is a small increase in risk and it would be valuable if this could be further studied in larger samples preferably with data on valproate dosage, trough levels and platelet counts. In one large single centre study, the risk of bleedings requiring acute decompression was shown not to be related to the surgical learning curve, and so, not to diminish over time. ${ }^{4}$ The importance of minimising such potentially lifethreatening complications during presurgical investigations cannot be overstated.

Five deaths directly related to subdural grid implantation were documented in the systematic review. ${ }^{13}$ This does not, however, cover complications related to depth electrodes. In general, complications have been reported to be less common with depth electrodes than with subdural grids, ${ }^{4} 5726-29$ but at least two fatalities have been reported with SEEG recordings. ${ }^{30} 31$ Also, Engel et al early on reported two deaths from intracerebral haemorrhage caused by depth electrodes. ${ }^{32}$

An important issue when striving to lower the risk of serious complications related to invasive monitoring is surveillance. It is clear from the literature that some reported deaths have been related to lack of $24 \mathrm{~h}$ professional surveillance. ${ }^{33}$ While serious complications cannot totally be avoided, their effects may be minimised with rapid intervention such as acute decompression. In our series, all complications were considered minor only because there were no permanent sequels. However, the most frequent complications were potentially dangerous subdural and epidural haematomas. The four Swedish centres in which subdural grids are used all have personalised $24 \mathrm{~h}$ surveillance of those patients and have, therefore, managed to act rapidly in case of complications.

The spectrum of definitions and scales makes it difficult to compare studies and, therefore, reporting only percentages is not very meaningful. The main issues are study methodology on the one hand, and the definitions of complications on the other. Most studies are single-centre retrospective studies, while a few studies analyse prospectively recorded data. ${ }^{92} 24$ It would be valuable if an agreement could be reached on how to report complications associated with invasive EEG monitoring, and agree about what is to be considered a complication and what could rather be described as a more or less expected adverse event. In the present study, we have not considered transient CSF leakage, fever and headache as complications, as all these commonly occur after implantation of intracranial electrodes. Neither have we had as a routine to report small asymptomatic subdural haematomas. We acknowledge that this is a limitation of the study, and are aware that it leads more to a 'top of the iceberg' reporting, but we capture symptomatic complications and especially all those necessitating interventions. Another limitation of the study is that the register does not contain information on the number of electrodes and duration of invasive monitoring, and these variables could, therefore, not be related to the risk of complications.

The main strength of the study is the prospective collection of population-based data in a large national series. Also, our external quality control ensures that the data has been correctly entered from the patient files into the register.

\section{CONCLUSION}

In this prospective population-based epilepsy surgery series, the most common complications were haematomas, and subdural grids carried the highest risk of complications. Close supervision and rapid interventions led to avoidance of permanent morbidity. Whether the slightly increased risk of haematomas with valproate treatment is clinically relevant needs further investigation, as does the finding of an increased risk for complications at epilepsy surgery for patients who had a complication during invasive monitoring.

There is a need for consensus on how to report complications related to invasive monitoring in order to provide patients with adequate information on risks.

Acknowledgements The authors thank Aldina Pivodic for help with the statistical analyses, and the steering committee of the Swedish National Epilepsy Surgery Register, and all the Swedish epilepsy surgery teams (in Gothenburg, Linköping, Lund, Stockholm, Umeå, Uppsala) whose contributions to the register are a prerequisite for national population-based studies. 
Contributors $\mathrm{EH}$ analysed the data and drafted the manuscript. JB analysed data, searched the literature and revised the manuscript. AE analysed data, performed the statistical analysis and revised the manuscript. BR is responsible for the acquisition of data, analysed data and revised the manuscript. RF is responsible for the acquisition of data, and revised the manuscript. KM designed the study, supervised the analysis and wrote the manuscript. All authors have approved the final version.

Funding The study was funded by grants from the Swedish Research Council grant number 521-2011-169 and the Sahlgrenska Academy at Gothenburg University through the LUA/ALF agreement grant number ALFGBG137431. The funding bodies had no role in the study design; in the collection, analysis and interpretation of data; in the writing of the report; and in the decision to submit the paper for publication.

\section{Competing interests None.}

Ethics approval The Regional Board of Medical Ethics at the University of Gothenburg.

Provenance and peer review Not commissioned; externally peer reviewed.

Open Access This is an Open Access article distributed in accordance with the Creative Commons Attribution Non Commercial (CC BY-NC 3.0) license, which permits others to distribute, remix, adapt, build upon this work non-commercially, and license their derivative works on different terms, provided the original work is properly cited and the use is non-commercial. See: http://creativecommons.org/ licenses/by-nc/3.0/

\section{REFERENCES}

1 Schuele SU, Luders HO. Intractable epilepsy: management and therapeutic alternatives. Lancet Neurol 2008;7:514-24.

2 Spencer S, Huh L. Outcomes of epilepsy surgery in adults and children. Lancet Neurol 2008;7:525-37.

3 Wiebe S, Blume WT, Girvin JP, et al. Effectiveness, efficiency of surgery for tempora lobe epilepsy study G. A randomized, controlled trial of surgery for temporal-lobe epilepsy. N Engl J Med 2001;345:311-18.

4 Wellmer J, von der Groeben F, Klarmann U, et al. Risks and benefits of invasive epilepsy surgery workup with implanted subdural and depth electrodes. Epilepsia 2012:53:1322-32.

5 Ross DA, Brunberg JA, Drury I, et al. Intracerebral depth electrode monitoring in partial epilepsy: the morbidity and efficacy of placement using magnetic resonance image-guided stereotactic surgery. Neurosurgery 1996;39:327-33; discussion 33-4.

6 Hamer HM, Morris HH, Mascha EJ, et al. Complications of invasive video-EEG monitoring with subdural grid electrodes. Neurology 2002;58:97-103.

7 Behrens E, Schramm J, Zentner J, etal. Surgical and neurological complications in a series of 708 epilepsysurgery procedures. Neurosurgery 1997;41:1-9; discussion-10.

8 Musleh W, Yassari R, Hecox K, et al. Low incidence of subdural grid-related complications in prolonged pediatric EEG monitoring. Pediatr Neurosurg 2006;42:284-7.

9 Blauwblomme T, Ternier J, Romero C, et al. Adverse events occurring during invasive electroencephalogram recordings in children. Neurosurgery 2011:69(2 Suppl Operative):169-75; discussion ons75.

10 Rydenhag B, Silander HC. Complications of epilepsy surgery after 654 procedures in Sweden, September 1990-1995: a multicenter study based on the Swedish National Epilepsy Surgery Register. Neurosurgery 2001;49:51-6; discussion 6-7.

11 Bekelis K, Radwan TA, Desai A, et al. Subdural interhemispheric grid electrodes for intracranial epilepsy monitoring: feasibility, safety, and utility: clinical article. J Neurosurg 2012:117:1182-8.

12 Burneo JG, Steven DA, McLachlan RS, et al. Morbidity associated with the use of intracranial electrodes for epilepsy surgery. Can J Neurol Sci 2006;33:223-7.
13 Arya R, Mangano FT, Horn PS, et al. Adverse events related to extraoperative invasive EEG monitoring with subdural grid electrodes: a systematic review and meta-analysis. Epilepsia 2013:54:828-39.

14 Nasreddine W, Beydoun A. Valproate-induced thrombocytopenia: a prospective monotherapy study. Epilepsia 2008:49:438-45.

15 Anderson GD, Lin YX, Berge C, et al. Absence of bleeding complications in patients undergoing cortical surgery while receiving valproate treatment. J Neurosurg 1997:87:252-6.

16 Ward MM, Barbaro NM, Laxer KD, et al. Preoperative valproate administration does not increase blood loss during temporal lobectomy. Epilepsia 1996;37:98-101

17 Bjellvi J, Edelvik A, Ekstedt G, et al. Complications of epilepsy surgery in Sweden 1996-2010: results from the Swedish National Epilepsy Surgery Register. Epilepsia 2012:53(Suppl 5):181.

18 Bauman JA, Feoli E, Romanelli P, et al. Multistage epilepsy surgery: safety, efficacy, and utility of a novel approach in pediatric extratemporal epilepsy. Neurosurgery 2005:56:318-34.

19 Onal C, Otsubo H, Araki T, et al. Complications of invasive subdural grid monitoring in children with epilepsy. J Neurosurg 2003;98:1017-26.

20 Swartz BE, Rich JR, Dwan PS, et al. The safety and efficacy of chronically implanted subdural electrodes: a prospective study. Surg Neurol 1996:46:87-93.

21 Simon SL, Telfeian A, Duhaime AC. Complications of invasive monitoring used in intractable pediatric epilepsy. Pediatr Neurosurg 2003;38:47-52.

22 Wiggins GC, Elisevich K, Smith BJ. Morbidity and infection in combined subdural grid and strip electrode investigation for intractable epilepsy. Epilepsy Res 1999:37:73-80

23 Korinek AM, Golmard JL, Elcheick A, et al. Risk factors for neurosurgical site infections after craniotomy: a critical reappraisal of antibiotic prophylaxis on 4,578 patients. J Neurosurg 2005;19:155-62.

24 Vale FL, Pollock G, Dionisio J, et al. Outcome and complications of chronically implanted subdural electrodes for the treatment of medically resistant epilepsy. Clin Neurol Neurosurg 2013;115:985-90.

25 Boviatsis EJ, Bouras TI, Kouyialis AT, et al. Impact of age on complications and outcome in meningioma surgery. Surg Neurol 2007:68:407-11; discussion 11.

26 Bekelis K, Desai A, Kotlyar A, et al. Occipitotemporal hippocampal depth electrodes in intracranial epilepsy monitoring: safety and utility. J Neurosurg 2013;118:345-52.

27 Fernandez G, Hufnagel A, Van Roost D, et al. Safety of intrahippocampal depth electrodes for presurgical evaluation of patients with intractable epilepsy. Epilepsia 1997:38:922-9.

28 Ozlen F, Asan Z, Tanriverdi T, et al. Surgical morbidity of invasive monitoring in epilepsy surgery: an experience from a single institution. Turk Neurosurg 2010;20:364-72.

29 Sansur CA, Frysinger RC, Pouratian N, et al. Incidence of symptomatic hemorrhage after stereotactic electrode placement. J Neurosurg 2007;107:998-1003.

30 Cossu M, Schiariti M, Francione S, et al. Stereoelectroencephalography in the presurgical evaluation of focal epilepsy in infancy and early childhood. J Neurosurg Pediatr 2012;9:290-300

31 Guenot M, Isnard J, Ryvlin P, et al. Neurophysiological monitoring for epilepsy surgery: the Talairach SEEG method. StereoElectroEncephaloGraphy. Indications, results, complications and therapeutic applications in a series of 100 consecutive cases. Stereotact Funct Neurosurg 2001;77:29-32.

32 Engel J Jr, Crandall P, Rausch R. The partial epilepsies. In: Rosenberg R, Grossman $\mathrm{R}$, Schochet $\mathrm{S}$, Heinz $\mathrm{E}$, Willis W, eds. The clinical neurosciences. New York: Churchill Livingstone, 1983:1349.

33 Wong $\mathrm{CH}$, Birkett J, Byth $\mathrm{K}$, et al. Risk factors for complications during intracranial electrode recording in presurgical evaluation of drug resistant partial epilepsy. Acta Neurochir (Wien) 2009:151:37-50. 